\title{
Rursus
}

Ruscus Poiétique, réception et réécriture des textes antiques

4 | 2009

Erotica

\section{L'Eroticos de Plutarque et les romans d'amour : échos et écarts}

\section{Michèle Biraud}

\section{(2) OpenEdition}

Journals

Édition électronique

URL : http://journals.openedition.org/rursus/250

DOI : $10.4000 /$ rursus. 250

ISSN : 1951-669X

\section{Éditeur}

Université Nice-Sophia Antipolis

Référence électronique

Michèle Biraud, «L'Eroticos de Plutarque et les romans d'amour : échos et écarts », Rursus [En ligne],

4 | 2009, mis en ligne le 12 février 2009, consulté le 19 avril 2019. URL : http://

journals.openedition.org/rursus/250 ; DOI : 10.4000/rursus.250

Ce document a été généré automatiquement le 19 avril 2019

Rursus 


\title{
L'Eroticos de Plutarque et les romans d'amour : échos et écarts
}

\author{
Michèle Biraud
}

1 Bon nombre de lecteurs de l'Eroticos de Plutarque ont dû être des lecteurs de romans tout autant que des lecteurs du Phèdre et du Banquet de Platon. Il est en effet bien établi maintenant que les lecteurs antiques du roman grec appartenaient au public cultivé et que ce genre était déjà développé au $\mathrm{I}^{\mathrm{er}}$ siècle. Si l'on considère, parmi les romans contemporains, celui de Chariton, il n'est pas impossible de le résumer comme une histoire d'amour contrariée par des péripéties qui étaient devenues conventionnelles depuis la comédie nouvelle, mais ce serait négliger les allusions aux classiques, dont l'identification fait découvrir un sens plus complexe de l'œuvre ${ }^{1}$, l'importance de l'analyse psychologique, qui se place dans la lignée de Thucydide, et les échos des exercices des écoles de rhétorique; tout ceci aurait été peu perceptible pour un lectorat peu cultivé2. Les personnages d'ailleurs se déclarent eux-mêmes pepaideumenoi, se font gloire de leur paideia, cette éducation à la grecque, à la fois conscience d'une culture propre et éthique de maitrise de soi et de respect d'autrui, qui les distingue des rustreset des barbares qui leur font subir des avanies, des épreuves qu'ils déclarent supporter précisément grâce à leur bonne éducation, à cette culture qu'ils ont intériorisée.

2 Plutarque n'évoque pas ce type de fiction, mais il exprime une prédilection pour les comédies de Ménandre comme littérature de divertissement qu'un intellectuel peut apprécier sans honte: "Quelle est la raison qui justifie vraiment qu'un homme cultivé aille au théatre, sinon le désir d'entendre Ménandre ? Y a-t-il d'autres occasions où l'on voit le théâtre se remplir de lettrés quand un personnage comique est mis en scène ? [...] De même que les peintres, quand ils ont la vue fatiguée, se tournent vers les couleurs des fleurs et des prairies, les philosophes et les gens qui s'adonnent aux études se reposent de leurs efforts soutenus et constants grâce à Ménandre qui permet à leur esprit de trouver, pour ainsi dire, une prairie fleurie, ombragée et parcourue par les brises $»^{3}$. Et il sait aussi que le goût de la philosophie vient à la lecture d'histoires qui sont l'illustration agréable d'une morale ou d'une pensée théorique : «Mais que parmi les ouvrages de philosophie, les jeunes lecteurs goûtent davantage ceux qui ne donnent pas l'impression d'être des 
écrits philosophiques et sérieux, qu'ils se montrent alors attentifs et conquis, c'est pour nous une évidence. Quand ils lisent en entier non pas seulement les Fables d'Esope, les résumés d'œuvres poétiques, l'Abaris d'Héraclide, le Lycon d'Ariston, mais aussi les théories sur l'âme mêlées de mythes, le plaisir les transporte »4.

Puisque les romans, ou du moins certains d'entre eux, ont été le délassement des esprits cultivés, que Plutarque lui-même a le goût de la récréation que représente pour lui la comédie de Ménandre, et qu'il sait bien que les récits qui contiennent une leçon cachée sont une bonne façon de capter l'attention d'un public qui n'est pas d'emblée intéressé par la philosophie, il n'y a rien d'étonnant à ce que Plutarque a choisi d'animer son traité philosophique en associant les discours théoriques sur l'amour à la relation d'une histoire d'amour particulièrement remarquable et racontée à la façon d'un roman.

Notre point de vue sera donc d'observer l'Eroticos avec le regard du lecteur de romans, pour apprécier les échos et les écarts par rapport à l'archétype du roman "première manière $»^{5}$, et l'utilisation que Plutarque fait des goûts et des compétences du lecteur de romans dans la création de sa propre œuvre.

5 A l'époque de Plutarque, se servir d'une narration fictive pour illustrer en action des conceptions théoriques était une possibilité acquise depuis longtemps pour les philosophes. En effet, le genre du roman philosophique avait été inauguré par la Cyropédie de Xénophon, et continué par des romans comme ceux de Iamboulos et Evhémère à l'époque hellénistique ${ }^{6}$. La trame générale de ces trois œuvres n'était pas une histoire d'amour, mais en revanche, c'est le cas pour un roman à peu près contemporain de l'œuvre de Plutarque, Les Merveilles d'au-delà de Thulé7.

Plutarque aime à entrecroiser une trame narrative romanesque avec le dialogue philosophique. A la différence de Platon, il ne se contente pas d'une situation de discours statique telle qu'une discussion de banquet ou une conversation sous un portique ou à l'ombre d'un platane : en même temps que se déroule le dialogue, certains personnages, désireux d'agir, agissent et modifient la situation initiale jusqu'à un renversement complet (ce qui est la définition même d'une séquence narrative). C'est le cas dans le Démon de Socrate, dont la trame narrative serait digne d'un roman. Le dialogue philosophique sur la démonologie y est enclavé dans le récit de la conspiration qui libéra (en 379) la ville de Thèbes de la domination spartiate. Récit et dialogue, entrelacés, y occupent chacun un volume égal. Le récit est une séquence narrative assez complexe: une situation initiale constituée de réunions répétées de gens d'esprit séditieux, sous couvert de philosophie (576B), est transformée par l'annonce d'un projet de retour des bannis pour assassiner les tyrans (576C) ; s'ensuit une dynamique d'actions constituée par l'attente impatiente des bannis, divers préparatifs et contre-temps, le rassemblement des conjurés chez Charon, d'ultimes péripéties qui manquent de peu de faire échouer l'entreprise (576D-596F); puis l'exécution des tyrans (597A-598C) rend possible l'instauration d'une nouvelle situation stable, celle du retour à la démocratie (598D-598F). Cette narration des manœuvres des conjurés et de l'assassinat des tyrans est, elle aussi, à rapprocher d'une littérature de type romanesque, celle des récits de complot, dont les périodes de tyrannie (règnes de Néron, Domitien ou Commode) ont, semble-t-il, été friandes, et de laquelle a survécu pour nous le roman par lettres de Chion d'Héraclée 8 .

7 Dans l'Eroticos, Plutarque réduit à presque rien le dialogue introductif (qui est une demande au fils de Plutarque de rapporter les propos tenus par son père et ses amis sur l'Hélicon à propos de l'Amour) et y traite par l'ironie les imitateurs du prologue du Phèdre qui jugent opportun de situer leurs dialogues philosophiques dans un paysage bucolique 
et essayent de rivaliser avec Platon dans la description de celui-ci [niveau 1 du tableau ciaprès]. Vient ensuite le véritable préambule [2.1], où sont exposées les raisons de la rencontre de Plutarque et des autres participants du dialogue. Il y est fait aussi mention des circonstances qui ont motivé ce dialogue, d'événements qui, comme le souligne l'un des interlocuteurs du prologue (749A), constituent un drama digne du théâtre, etqui suscitent le pathos. C'est la narration de ces événements [niveau 3] qui constitue, avec la relation des déplacements de Plutarque et ses amis [niveau 2], le double cadre narratif englobant des propos philosophiques [niveau 4] et le terreau dans lequel ils s'enracinent.

\begin{tabular}{|c|c|c|c|}
\hline 1. & Dialogue introductif & $\begin{array}{l}748 \mathrm{~B} \\
\mathrm{749 \textrm {A }}\end{array}$ & $-0,5 p$ \\
\hline 2.1. & $\begin{array}{l}\text { Exposé des raisons de Plutarque de venir à Thespies au moment des fêtes } \\
\text { d'Eros, puis d'aller sur l'Hélicon avec ses amis, et parmi eux deux habitants } \\
\text { de Thespies, Anthémion (cousin de Bacchon) et Pisias (l'un des érastes de } \\
\text { Bacchon) }\end{array}$ & 7498 & $-0,3 p$ \\
\hline 3.1. & Début du récit des amours d'Isménodore et de Bacchon & 7490 & $-0,6 p$ \\
\hline 4.1 & $\begin{array}{l}\text { Discussion générale : comparaison de l'amour des garçons et de l'amour } \\
\text { des femmes }\end{array}$ & $\begin{array}{l}7501 \\
752 \mathrm{D}\end{array}$ & $2,5 \mathrm{p}$ \\
\hline 4.2. & Discussion particulière : Bacchon doit-il épouser Isménodore? & $754 \mathrm{D}$ & $-2 p$ \\
\hline 2.2. & Arrivée d'un messager (ami de Pisias) & $754 \mathrm{H}$ & $0,15 p$ \\
\hline 3.2. & Récit du messager : l'enlèvement de Bacchon par Isménodore & $\begin{array}{c}754 \mathrm{H} \\
755 \mathrm{~B}\end{array}$ & $-0,5 p$ \\
\hline 2.3. & Pisias et Protogène, indignés, rentrent à Thespies. & $755 \mathrm{C}$ & $-0,3 p$ \\
\hline 4.3. & Commentaires sur le comportement d'Isménodore & $756 \mathrm{~A}$ & $-1 p$ \\
\hline 2.4. & Un second messager (ami d'Isménodore) vient chercher Anthémion & $756 \mathrm{~A}$ & $0,1 p$ \\
\hline 3.3. & $\begin{array}{l}\text { Récit du messager : l'un des gymnasiarques veut faire opposition à cet } \\
\text { enlèvement }\end{array}$ & $756 \mathrm{~A}$ & $0,1 p$ \\
\hline 4.4. & $\begin{array}{l}\text { Débat de Plutarque avec ses amis sur l'Amour et la supériorité de l'amour } \\
\text { conjugal }\end{array}$ & $\begin{array}{l}756 \mathrm{~B} \\
771 \mathrm{C}\end{array}$ & $-16 p$ \\
\hline 2.5 & $\begin{array}{l}\text { Plutarque et ses amis qui, tout en parlant, avaient repris le chemin de } \\
\text { Thespies, rencontrent un troisième messager, ami de Pisias }\end{array}$ & 7711 & $0,1 p$ \\
\hline 3.4 & $\begin{array}{l}\text { Récit du messager: Pisias a cessé de s'opposer au mariage; c'est même lui } \\
\text { qui mène les préparatifs du mariage }\end{array}$ & 7710 & $0,1 p$ \\
\hline
\end{tabular}


Sur les vingt-trois pages de l'édition de référence (c'est-à-dire sans compter l'espace nonquantifiable de la lacune en $766 \mathrm{D}$ ), la relation des allées et venues de Plutarque et de ses amis et des rencontres qu'il fait $[2.1,2.2,2.3,2.4,2.5]$ occupe à peu près une page et demi ; quant à la séquence narrative formée par l'histoire d'amour d'Isménodore [3.1], l'enlèvement de Bacchon [3.2], l'opposition que cela suscite dans l'opinion [3.3] et les préparatifs du mariage [3.4.], elle n'occupe guère qu'une page et demi elle aussi. Près de $85 \%$ du texte est consacré aux dialogues, mais environ un cinquième du temps de dialogue est consacré à commenter et à expliquer le comportement d'Isménodore et la situation de Bacchon, ce qui confère à la fable amoureuse une part évidemment beaucoup plus importante dans la mémoire du lecteur que le peu de pages de narration suivie qui lui sont consacrées.

Plutarque a greffé ses propos philosophiques sur une narration d'affaire amoureuse dont nous venons de voir, en analysant la structure de l'œuvre, qu'il lui a accordé une place englobante par rapport au dialogue philosophique. Elle lui sert en quelque sorte d'introduction et de conclusion [3.1 et 3.4]. Et il y a deux autres brefs affleurements de cette narration [3.2 et 3.3] qui viennent interrompre le dialogue (à la fin du premier quart et du premier tiers de l'œuvre), la première fois pour permettre au débat de rebondir avec des commentaires sur l'intérêt d'avoir pour compagne une femme telle qu'Isménodore, et la seconde fois, pour lancer le débat proprement philosophique.

De plus, cette narration d'histoire d'amour intervient en harmonie avec les développements théoriques sur l'amour et le mariage. Ainsi, le récit de l'enlèvement intervient après que Plutarque (le personnage) ait fait ressortir l'intérêt d'un mariage avec une femme socialement plus expérimentée (754 C-D); on voit alors (754 E-F) Isménodore se servir de son réseau de relations pour comploter et réussir l'enlèvement. Lors des commentaires sur le rapt, Anthémion déclare qu'en enlevant Bacchon,

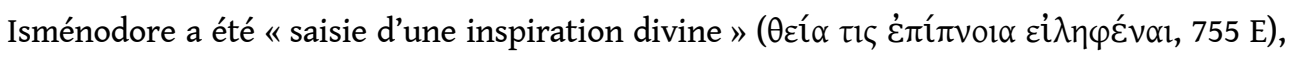
ce qui permet de lancer le débat sur l'identité divine d'Eros (qui commence en 756B). De sorte qu'inversement, au terme de l'ultime apologie de l'amour conjugal, on ne peut envisager, au niveau du récit, que le mariage d'Isménodore et de Bacchon. De ce point de vue, le récit est une illustration exemplaire de la pensée développée dans les dialogues. La différence avec l'exemplum rhétorique traditionnel est que celui-ci est inclus dans le discours ${ }^{9}$, alors qu'ici ce sont les discours qui sont inclus dans la trame narrative: la fiction amoureuse a commencé avant le début du dialogue, dont l'existence est présentée comme essai d'évaluation de cette situation; elle resurgit par épisodes, et elle achève l'ouvrage.

11 Par ailleurs, comme les péripéties de l'affaire amoureuse, au lieu d'être racontées en détail, ont lieu, pour la plupart en coulisses, et viennent seulement par moments au premier plan, comme par un eccyclème, à la faveur de la relation des faits par l'un des personnages du dialogue, le temps de la fiction amoureuse est le même que le temps de l'énonciation du dialogue, et même le déborde en amont, ce qui renforce l'effet englobant de la fiction par rapport au dialogue.

12 La structure de la fable amoureuse, qui constitue une séquence narrative complète ${ }^{10}$, est celle des débuts de romans d'amour et d'aventure contemporains de l'Eroticos ${ }^{11}$. Les deux amoureux sont beaux et bien nés, leur situation familiale ou sociale crée quelque obstacle 
à leur union, mais ils manifestent leur sentiment avec persévérance, un adjuvant se manifeste, la foule finit par applaudir à leur amour et une noce publique est célébrée.

Détaillons maintenant ces échos directs des romans. Ils sont nombreux, en particulier dans le premier passage de récit (749D - 750A) et dans l'épilogue (771D) :

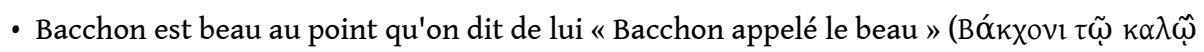

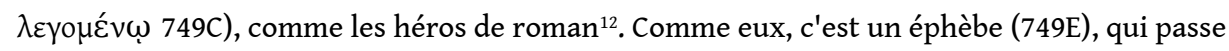
ses journées au gymnase $(754 \mathrm{E})^{13}$ et qui est plein de qualités morales ${ }^{14}$. Il est de bonne naissance, quoique son origine soit un peu moins illustre que celle de la jeune femme (749DE) ${ }^{15}$.

- L'obstacle au bonheur des amoureux provient, comme dans les romans, de l'opposition de la famille (749E : inquiétudes de la mère de Bacchon $)^{16}$ et des prétendants $(749 \mathrm{~F}: \text { Pisias })^{17}$.

- Le plus sûr adjuvant, chez Plutarque, est un cousin du jeune homme, Anthémion $(749 \mathrm{E})^{18}$.

- L'importance de la foule dans l'évolution et le dénouement des affaires de cœur est tout aussi importante ici que dans les romans : curieuse de ce qui arrive aux notables ${ }^{19}$, elle exerce une fonction de commentaire $(755 \mathrm{~A}-\mathrm{B})^{20}$ et surtout d'agent qui presse la décision $(756 \mathrm{~A})^{21}$. Au dénouement, comme dans les romans, le mariage, avec son cortège nuptial, intéresse toute la ville ${ }^{22}$. Et, ultime trait de parallélisme, la cérémonie se déroule publiquement dans un temple (771D) ${ }^{23}$.

14 Mais sur ce fond commun de topoï qui ne concerne que les arrière-plans de la fiction, le narrateur construit son personnage principal, Isménodore, en privilégiant des traits de comportement ou de situation sociale qui ne sont pas ceux des héroïnes de roman, même s'ils sont parfois des variantes attestées pour des personnages secondaires.

Ainsi, la naissance de son amour n'est pas présentée de la façon habituelle pour les héros de roman, le coup de foudre au premier regard (alors que c'est cette première surprise de l'amour, dans son ingénuité, qui semble intéressante à décrire pour les romanciers), mais Plutarque utilise néanmoins deux topoï qui conditionnent la naissance du sentiment amoureux chez certains personnages secondaires des romans : l'importance des éloges d'autrui, avant même le premier regard ${ }^{24}$, et l'inclination progressive résultant de la fréquentation ${ }^{25}$. Cette variation est nécessaire au projet de Plutarque qui est de montrer que l'amour conjugal, contrairement à ce qu'affirment alors les détracteurs de l'amour des femmes, n'est pas seulement sensuel. Or l'amour au premier regard ne peut être provoqué que par la vision d'une belle apparence tandis que l'amour par inclination progressive naît de la découverte des qualités d'esprit et de cœur de l'être aimé. De plus, cet amour, pour être raisonné, n'en est pas moins puissant et capable de toutes les audaces, comme le narrateur le montre par le comportement passionné d'Isménodore qui fait enlever celui qu'elle aime.

D'autre part, cette jeune femme est une veuve. Ce choix de statut social est en rupture totale avec les usages des romans, et d'ailleurs aussi avec les usages de l'époque, où l'on considérait une veuve amoureuse comme quelqu'un qui ne sait pas maîtriser ses sens et se rend ridicule ${ }^{26}$. En effet, dans la société gréco-romaine du $\mathrm{I}^{\mathrm{er}}$ siècle de notre ère, une jeune veuve est bien considérée si elle mène une vie discrète et rangée, reste veuve quelques années pour élever ses enfants et se remarie ensuite à un homme au moins aussi âgé qu'elle, parce qu'il est convenable et utile à sa santé qu'une femme encore jeune soit mariée, mais sans qu'il soit approprié de le justifier par un quelconque sentiment amoureux. Le propos de Pisias «Les femmes honnêtes ne doivent pas s'éprendre des hommes ( $(\dot{\varepsilon} \rho \tilde{\alpha} v)$ ni accueillir leur amour ( $\dot{\varepsilon} \rho \tilde{\alpha} \sigma \theta \alpha l) »(752 C)$ trouve son parallèle dans l'histoire du mariage d'Apulée avec une veuve, telle qu'il la rapporte lui-même dans son 
Apologie (pro se de magia libro) : accusé par le fils d'une veuve, au comportement jusque là irréprochable, de l'avoir ensorcelée pour la décider à l'épouser, Apulée produit une lettre de cette femme à son fils, où elle réfute cette allégation en ces termes: «je ne suis pas

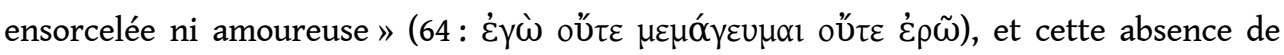
passion est considérée comme un signe de sa vertu et de sa lucidité. L'union avec une veuve ne peut être qu'un moyen pour un homme peu scrupuleux de s'enrichir ${ }^{27}$.

C'est pourquoi Plutarque prend bien soin de démarquer son Isménodore de ce genre de personnage, en insistant sur sa naissance illustre (749D, 752E), en la présentant comme une femme attirante ${ }^{28}$ et jusque là réservée ${ }^{29}$, et en s'attachant à montrer que sa passion, qui s'est développée progressivement ${ }^{30}$ en apprenant à mieux connaître le fils de son amie, est une inclination sensée quoiqu'elle soit excessive. Il joue des différences et des ressemblances de son personnage avec la veuve âgée et la jeune fille pour retenir de l'une son statut social et économique et de l'autre ses caractéristiques physiques et morales, et il construit ainsi un personnage sympathique à défaut d'être d'une vraisemblance conventionnelle.

En effet, la relation entre Isménodore et Bacchon est différente de la relation à égalité qui se noue entre les jeunes gens protagonistes des romans (qui sont tous deux à la fin de l'adolescence, éprouvent un coup de foudre réciproque et des symptômes identiques du mal d'amour). Bien plus, elle se calque sur celle des couples homosexuels masculins, qui est une relation inégale où un éraste, plus âgé que son éromène, aime avec passion cet adolescent dont le rôle reste passif ${ }^{31}$. Isménodore est plus âgée que le jeune homme ${ }^{32}$, et même les âges sont inversés, comme on le souligne en 753A et 764D; c'est elle qui déclare son amour ${ }^{33}$, c'est elle qui enlève le jeune homme ${ }^{34}$. Bref son âge et ses actions sont ceux de l'éraste, d'un homme passionné, ils sont aux antipodes de ceux d'une future épousée ${ }^{35}$. C'est pourquoi Pisias peut la tourner en ridicule en suggérant qu'elle devrait aller jouer la sérénade sous les fenêtres de Bacchon, comme les galants de roman ou d'élégie sous la fenêtre de leur aimé(e) (753B), et finit par constater que la situation est typique du monde à l'envers : « il faut remettre aux femmes le gymnase et le bouleutérion » $(755 \mathrm{C})^{36}$. Et après l'enlèvement, le narrateur aussi s'amuse à souligner l'inversion des rôles : c'est au jeune homme qu'on fait la traditionnelle ${ }^{37}$ « toilette de la mariée » (755A).

Toutefois, bien que l'auteur se joue de la norme sociale telle qu'elle est idéalisée dans les romans, il crée avec Isménodore un nouveau personnage qui serait plutôt hyperromanesque qu'anti-romanesque, en ce sens qu'il est conçu pour susciter l'étonnement. Cette intention d'étonner rapproche Plutarque des romanciers dans leur activité commune de créateurs de personnages. Cette intention est tout à fait explicite puisqu'il utilise l'épithète de paradoxos pour qualifier, dès l'exposé de la situation initiale, le projet de mariage envisagé par Isménodore (749E), et le terme de thauma pour désigner, un peu plus loin, l'enlèvement du jeune homme (745E). Or toutes les héroïnes de roman ont un comportement surprenant dans des situations extrêmes. Anthia a plus d'une fois l'occasion de préférer mourir plutôt que de cesser d'être chaste et fidèle, et c'est le hasard de circonstances invraisemblables qui, à chaque fois, lui sauve la vie. Quant à Callirhoé, Chariton réalise le tour de force d'en faire une épouse bigame mais néanmoins vertueuse et fidèle à son premier époux, ce qui est totalement contraire à toutes les idées reçues, et cela par un enchaînement astucieux de situations et grâce aux détours d'une analyse psychologique relativement complexe, et il dévoile son intention d'associer paradoxalement bigamie et fidélité en présentant son héroïne associée, par allusions, aux figures mythiques d'Ariane, d'Hélène et de Pénélope. Somme toute, Isménodore n'est ni 
plus extrême qu'Anthia ni plus paradoxale que Callirhoé. Par le caractère anormal et sans précédent de ses sentiments et de son comportement, on peut donc considérer qu'elle intègre le paradigme des héroïnes de roman.

Dans l'élaboration du personnage d'Isménodore et de son aventure, Plutarque joue donc des contradictions du genre romanesque, qui conserve les usages sociaux conventionnels et les stéréotypes narratifs, mais a le besoin de renouveler l'intérêt des lecteurs par la recherche de l'extraordinaire. Par le jeu des échos et des écarts, Plutarque dessine une figure féminine originale, qui, à force d'être paradoxale, s'avère à la fois anti-romanesque et hyper-romanesque, et donc tout à fait propre à susciter un débat sur l'amour, lequel vient s'entrelacer avec naturel et vraisemblance au canevas narratif.

Cet exemplum conçu pour frapper l'esprit du lecteur trouve chez Plutarque un nouveau statut, non plus local mais englobant ${ }^{38}$. Cette nouvelle perspective discursive, et le choix du genre narratif alors émergent, le roman, favorisent l'utilisation de la fiction des amours d'Isménodore comme support de mémorisation des arguments philosophiques et moraux développés dans le dialogue qui sont favorables à l'amour au sein du couple légitime.

\section{NOTES}

1. Voir notre article «L'hypotexte homérique et les rôles amoureux de Callirhoé dans le roman de Chariton ", in Sémiologie de l'amour dans les civilisations méditerranéennes, Publications de la Faculté des Lettres de l'Université de Nice, 29, 1985, p. 21-27.

2. Voir E.L. Bowie, « Les lecteurs du roman grec ", Le monde du roman grec, 1992, Paris, P.E.N.S., p. 55-62.

3. Comparaison d'Aristophane et de Ménandre, $854 \mathrm{~A}-\mathrm{C}$.

4. Comment le jeune garçon doit lire les poèmes, 14E, trad. A. Philippon, CUF.

5. Celui du ${ }^{\mathrm{er}}$ siècle, selon la classification de N. Holzberg (Der antike Roman, München und Zurich, 1986).

6. D'après les résumés de Diodore de Sicile, Bibliothèque historique, II, 55-60 (pour celui de Iamboulos) et $\mathrm{V}, 41-46$ (pour celui d'Evhémère), il semble que, dans le cadre d'un roman d'aventure et d'un voyage fabuleux, aient été développées des utopies sociales et politiques et certaines conceptions d'ordre religieux.

7. Ce roman d'Antonius Diogénès est connu par un résumé dans la Bibliothèque de Photius (II, 166), une citation dans la Vie de Pythagore par Porphyre et peut-être un fragment papyrologique. Il fut écrit au premier ou au deuxième siècle de notre ère. On le considère généralement comme un roman d'inspiration pythagoricienne, mais G.Anderson (Eros sophistes, Ancient novelists at play, American Classical Studies $\left.n^{\circ} 9,1982\right)$ y voit une parodie de roman géographico-ethnophilosophique. L'intrigue se résume succinctement à ceci : alors que Dinias part réaliser, en savant curieux, un tour du monde de la Mer Extérieure, Dercyllis, une jeune fille dont les parents ont été plongés par Paapis dans un sommeil qui passe pour la mort, doit elle aussi quitter sa patrie et entreprendre un long voyage à la poursuite de ce mage égyptien, périple qui finit par la mener à Thulé, où elle rencontre Dinias, et subit bien des épreuves, avant qu'ils ne puissent retourner à Tyr, chacun de son côté, et finir par se marier. 
8. Cette œuvre, qui raconte comment Chion, qui était venu à Athènes pour suivre l'enseignement de Platon, retourne dans sa patrie, Héraclée, pour tuer le tyran Cléarque, est considérée comme contemporaine de la tyrannie de Domitien (A. Billault, «Les lettres de Chion d'Héraclée ", R.E.G., 1977, XC, p. 29-37, B. Zucchelli, « A proposito dell'epistolario di Chione d'Eraclea », Paideia, 1986, XLI, p. 13-24). Editions: I. Düring, Chion of Heraclea : a novel in letters, Göteborg (Acta Univ. Goteburg), 1951, P.L. Malosse, Lettres de Chion d'Héraclée (texte révisé, traduction et commentaire), Salerne (Helios), 2004. Deux autres études littéraires importantes sur cette œuvre : D. Konstan et M. Philip, «Chion of Heraclea : a philosophical novel in letters », Apeiron, 1990, XXIII, 4, p. 257-279, P. A. Rosenmeyer, «The epistolary novel », in Greek fiction: the greek novel in context,J.R. Morgan \& R. Stoneman eds., 1994, London : Routledge, p. 146-165.

9. L'Eroticos offre des exemples de ce type, qui sont historiques $(768 \mathrm{~B}, 770 \mathrm{D})$ ou considérés comme tels (Sémiramis, 753D).

10. Focalisée sur l'idée directrice "Isménodore veut obtenir Bacchon", elle présente l'organisation canonique suivante: Etat initial: attirance croissante [3.1]; Force transformationnelle : l'enlèvement [3.2]; Dynamique : complots des adjuvants et des opposants [3.3] ; Force équilibrante : la réconciliation autour de la décision du mariage [3.4].

11. On entendra évidemment ici par "romans" les romans grecs qui sont de l'époque de Plutarque, ou antérieurs. Si l'on suppose que l'Eroticos a été écrit entre 100 et 120, cela correspond à la période du roman " première manière » selon la classification de N. Holzberg, c'est-à-dire un roman d'amour de tonalité sérieuse, idéalisant et sentimental, dont le schéma narratif est constant : rencontre fortuite du jeune homme et de la jeune fille, coup de foudre réciproque, découverte de la souffrance d'aimer sans espoir car cette union n'était pas dans les projets des parents, enfin mariage puis voyage semé d'épreuves (tempête, pirates, séparation, esclavage, tentatives de séduction...) ; le roman se termine par les retrouvailles et le bonheur final. Nous connaissons de ce type de roman les quelques pages du roman de Ninos, et le Chairéas et Callirhoé de Chariton, qui sont antérieurs ou contemporains à l'œuvre de Plutarque ; le roman de Xénophon d'Ephèse, Habrocomès et Anthia, en relève aussi par sa composition et sa thématique, bien que sa première attestation papyrologique soit seulement de la fin du II ${ }^{\text {ème }}$ siècle (Ce roman n'est probablement ni antérieur au règne d'Hadrien - au cours duquel on a relevé la première mention de l'irénarque de Cilicie - ni postérieur à la mort de Commode - 192 - car l'auteur décrit un Orient exempt de guerres civiles). Dans un deuxième temps, dont les œuvres attestées, celle d'Achille Tatius et celle d'Apulée, datent de la deuxième moitié du II ${ }^{\text {ème }}$ siècle, le roman prend un recul ironique par rapport aux premières productions du genre; j'ai cependant parfois cité Achille Tatius, car il reprend beaucoup de traits du roman "première manière », avant de les subvertir.

12. Dans le roman de Xénophon d'Ephèse, Habrocomès, qui est qualifié de «chef d'œuvre de

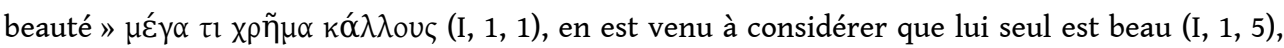
qu'il est plus beau même qu'Eros (I, 1, 6) - cette hubris va susciter les aventures, dues au désir de vengeance d'Eros. Le Chairéas de Chariton est aussi un "jeune homme de belle apparence »

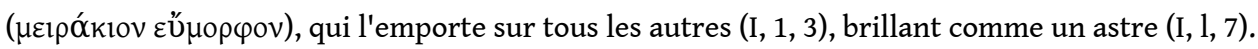

13. Comme le héros de Chariton: "alors Chairéas quittait les exercices du gymnase » $(I, 1,7)$; voir aussi Xénophon d'Ephèse, I, 1, 2. La fréquentation du gymnase est un signe de bonne éducation, à la différence des relations avec les courtisanes et de la pratique des jeux de hasard.

14. Le héros de Xénophon d'Ephèse ( $(, 1,2)$ possède « toutes les qualités de l'âme » ( $\tau \grave{\alpha} \tau \tilde{\eta} \varsigma ~ \psi v x \tilde{n} \varsigma$ $\alpha \gamma \alpha \theta \alpha ́)$; la paideia est une qualité des héros chez Chariton et Xénophon d'Ephèse.

15. Dans le roman de Chariton, Callirhoé est la fille d'Hermocrate, le stratège de Syracuse alors que le père de Chairéas est « le deuxième personnage de Syracuse » $(\mathrm{I}, 1,2)$.

16. Dans le roman de Chariton (I, 1, 9), le père du héros constate: «Hermocrate ne saurait te donner sa fille » et déconseille à son fils de faire une demande de mariage vouée à l'échec : «tu 
nous éviteras ainsi de nous faire outrager ». Dans celui d'Achille Tatius, le père du jeune homme lui a choisi pour fiancée une jeune fille différente de celle dont il est épris.

17. Dans le roman de Chariton (I, 2-4), il y a un complot des prétendants évincés de Callirhoé contre le bonheur du jeune couple qui joue un rôle très important dans le développement de l'intrigue romanesque.

18. Il en va de même dans le roman d'Achille Tatius, où Clinias, un peu plus âgé et plus expérimenté que le jeune héros, lui prodigue sesconseils et l'aide dans sa fuite; dans leroman de Chariton, c'est un ami du jeune homme, Polycharme, qui tient ce rôle.

19. Dans le roman, elle a une fonction de badaud et elle est constamment admirative : tantôt elle croit voir dans les héros des divinités (Xénophon d'Ephèse I, 12, 1-2; V, 13), tantôt elle fait cortège aux héros lors de leur départ (Xénophon d'Ephèse I, 10,6), tantôt elle veut savoir toute l'aventure (Chariton, VIII, 7, 1-3).

20. Innombrables sont les mentions de la phêmê et de la doxa dans les romans. En particulier, on voit la foule se diviser en deux partis lors du procès de Babylone (Chariton, VI, 1, 2: « La ville <de Babylone> était séparée en deux camps : les partisans de Chairéas disaient ' - ', ceux de Dionysios répliquaient " - '»).

21. Le début du roman de Chariton offre un parallèle, lorsque Chairéas dépérit d'amour pour la fille d'Hermocrate, qui ne souhaite pas s'allier avec sa famille (Chariton I, 1, 11) : « Le peuple prit place à l'assemblée et ne fit que crier sans arrêt : 'Bel Hermocrate, grand général, sauve Chairéas. Ce sera là ton principal titre de gloire. La cité souhaite aujourd'hui ce mariage de deux époux dignes l'un de l'autre' ». " Le peuple tout entier bondit hors du théâtre : les jeunes allaient chez Chairéas, les magistrats faisaient cortège à Hermocrate et il $\mathrm{y}$ avait aussi les femmes pour conduire la fiancée. On chantait l'hyménée par toute la ville; les rues étaient remplies de couronnes, de flambeaux... » $(\mathrm{I}, 1,12)$.

22. «Dès l'aube la ville était ornée de couronnes, chacun offrait des sacrifices devant sa propre maison» (Chariton, III, 2, 15). «Ephèse n'était que festins et couronnes, il n'était bruit partout que des noces qui se préparaient » (Xénophon d'Ephèse, I, 7, 3) ; il s'ensuit même une fête de nuit $(\mathrm{I}, 8,1)$.

23. «La foule se rassembla en masse autour du temple de la Concorde où traditionnellement les fiancées étaient conduites à leurs maris » (Chariton, III, 2, 16).

24. En 749D, Isménodore entend tenir des propos favorables sur le jeune homme. Cette situation s'observe dans le roman de Xénophon d'Ephèse en 1, 2, 9 («rapidement vint aux oreilles de chacun l'opinion qu'on avait de l'autre, et chacun avait le désir de voir l'autre ») et dans celui d'Achille Tatius, en II, 13, 1, où Callisthénès tombe amoureux par ouï-dire.

25. En $749 \mathrm{D}:$ « du fait des fréquentes occasions qu'elle avait de le rencontrer et de lui parler, elle

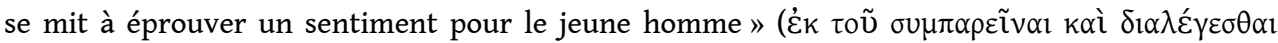

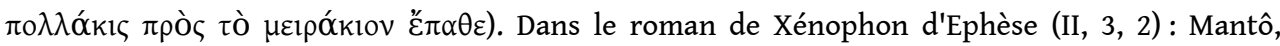
« vivant continuellement près d'Habrocomès », finit par s'éprendre de lui.

26. Voir le conte milésien de la Matrone d'Ephèse, raconté par Phèdre (Mulier uidua et miles, App. 13) et par Pétrone dans le Satiricon. Il faut arriver aux œuvres plus distanciées de la fin du lle siècle pour trouver dans le roman d'Achille Tatius un personnage de veuve agréable et assez sympathique (jeune, belle, riche, dévouée à ses amis), encore que son obsession amoureuse soit considérée comme une maladie qui fait pitié au héros, et devait faire sourire les lecteurs ; mais, dans ce cas, Plutarque a peut-être été une source d'inspiration pour Achille Tatius.

27. C'est ce dont on soupçonne Apulée (66sq). Dans le roman de Xénophon d'Ephèse, c'est précisément ce que fait un des personnages secondaires, un ancien brigand : "A Tauroménion, Hippothoos commença par mener une vie de misère. Mais un beau jour une vieille s'éprit de lui

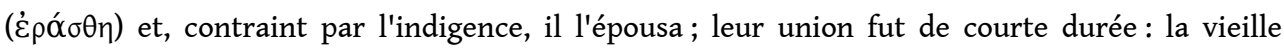
mourut, le laissant riche et opulent ». $(\mathrm{V}, 9,1)$. Juridiquement, en effet, la veuve est libre, elle a 
pu hériter la fortune de son mari s'il a fait un testament en ce sens, elle peut la transmettre ellemême à qui elle veut.

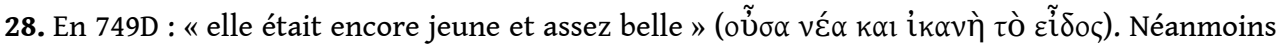
elle n'est pas la plus belle de toutes, à la différence des jeunes héroïnes de roman : Callirhoé est

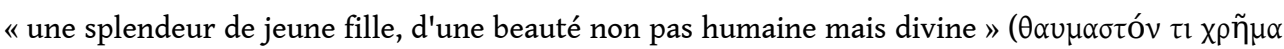

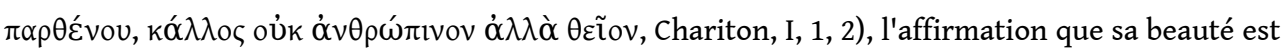
plus qu'humaine est réitérée maintes fois; il en est de même pour l'Anthia de Xénophon d'Ephèse (I, 2, 5-7 et passim).



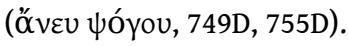

30. C'est là une divergence avec les coups de foudre des héros de romans. Voir par exemple, dans le roman de Chariton (I, 1, 6), comment les deux héros, se rencontrant au détour d'une rue, « sans

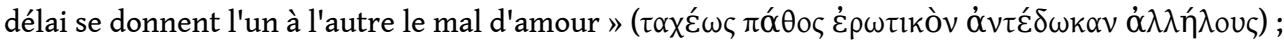
aussi en Xénophon d'Ephèse, I, 3. Néanmoins, la jeune Mantô du roman de Xénophon d'Ephèse, fille d'un brigand qui retient le héros prisonnier, est un autre exemple d'inclination progressive ; le narrateur juge utile de l'expliquer par le fait qu'elle "vivait constamment près d'Habrocomès » (II, 3, 2).

31. Lorsqu'il y a des aventures homosexuelles dans les romans, ce sont seulement celles des seconds rôles masculins. Dans le roman de Xénophon d'Ephèse, le brigand Hippothoos a deux amants successifs. Dans le roman d'Achille Tatius, le cousin du héros, Clinias, vit une relation homosexuelle fondée sur le désir (I, 7 et 12-14), et les héros rencontrent Ménélas, qui leur raconte la véritable passion qu'il a éprouvée pour un jeune homme (II, 34).

32. Voir 749E, 753A, 754D. En revanche, les couples d'amoureux des romans sont très jeunes, et sensiblement du même âge (voir les premières pages des romans de Chariton et Xénophon d'Ephèse).

33. Bien loin d'aller déclarer son amour, la jeune héroïne des romans n'ose pas en parler, même à ses parents : "plus terrible était la souffrance de la jeune fille à cause de son silence, dû à sa honte d'être découverte » (Chariton I, 1, 8) ; Anthia reste silencieuse (Xénophon d'Ephèse, I, 3, 2 ; I, 4,6$)$; dans sa lettre à Habrocomès, où elle lui avoue son amour, Manto estime que cette déclaration « est peut-être inconvenante pour une vierge, mais nécessaire pour une femme qui aime » (Xénophon d'Ephèse, II, 5, 1).

34. L'enlèvement est un topos du roman grec, mais jamais ce n'est une femme qui enlève un jeune homme : tantôt l'amoureux enlève la jeune fille consentante (Clitophon enlève Leucippé [Achille Tatius, II, 31], Aigialeus, à Sparte, enlève Thelxiné [Xénophon d'Ephèse, V, 1, 5-8]), tantôt il enlève une jeune fille malgré elle (Callisthénès enlève Calligoné [Achille Tatius, II, 16, 2]); ailleurs, l'éraste enlève l'éromène (Hippothoos enlève Hyperanthès [Xénophon d'Ephèse, III, 2, 10-11]).

35. Une femme peut avoir un comportement viril pour défendre ou venger un père (Antigone, Electre), un frère (Antigone, Iphigénie dans l'Iphigénie en Tauride), un mari (voir l'Hélène d'Euripide), ou pour se sacrifier pour son époux (Alceste) ou pour la patrie (Macarie). Plutarque a d'ailleurs réuni dans un traité intitulé Mulierum uirtutes des exemples de ce type de comportement. Mais nulle part aucune femme convenable ne commet d'actes inspirés par la passion amoureuse ; la seule référence possible est celle de Sappho (exploitée en 763A).

36. Qui restaient deux lieux exclusivement virils.

37. Autre topos romanesque : "après que ses servantes l'eurent rapidement parée... » (Chariton, I, 1, 15 ; voir aussi Chariton, III, 2, 16).

38. Dans le Discours Euboïque de Dion, la longue narration, d'une élaboration digne d'un roman, reste enclavée dans le discours, de façon traditionnelle. 


\section{RÉSUMÉS}

Dans ses dialogues philosophiques, Plutarque confère au récit une place considérable: non seulement il en fait une illustration exemplaire de la pensée philosophique (dans l'Eroticos, la fiction d'aventure amoureuse est en harmonie avec les développements théoriques sur l'amour et le mariage) mais il entrecroise la trame narrative avec les développements théoriques, le dialogue étant contemporain des événements racontés et en partie suscité par eux. La fable amoureuse de l'Eroticos a la même structure que les débuts de romans d'amour contemporains, et présente aussi de nombreux échos avec eux dans la définition des rôles actantiels des personnages ; son principal personnage est une femme passionnée, excessive et paradoxale à l'égal des héroïnes de Chariton et de Xénophon d'Éphèse, mais au point même de renverser les stéréotypes conventionnels du roman en ce qu'elle est l'éraste du couple, et de ce fait un exemplum adéquat dans le genre du dialogue philosophique sur l'amour.

In his philosophical dialogues, Plutarque confers a considerable place to the narrative: not only he makes of it an exemplary illustration of the philosophical thought (in Eroticos, the fiction of loving adventure is in accordance with the theoretical developments on love and marriage) but he crosses also the narrative texture with the theoretical developments, the dialogue being contemporary of the related events and partially aroused by them. The love story of the Eroticos has the same structure as the beginning of the contemporary love novels, and also presents numerous echos with them concerning the definition of the actantial roles of the characters; his main character is a passionate, excessive and paradoxical woman just like the heroines of Chariton and Xenophon of Ephesus, but to such an extent that it knocks down the conventional stereotypes of the novel because she is the erastes of the couple, and so an adequate exemplum in the genre of philosophical dialogue about love.

\section{INDEX}

Keywords : Eroticos, Greek novel, Love, Marriage, Paradox, Philosophical dialogues, Plutarchus, Women

Mots-clés : amour, dialogue philosophique, Eroticos, femmes, mariage, paradoxe, Plutarque, Roman grec

\section{AUTEUR}

\section{MICHĖLE BIRAUD}

BCL, CNRS, Université de Nice 